\title{
OH PLIF Visualization of the UVa Supersonic Combustion Experiment: Configuration A
}

\author{
C.T. Johansen", C.D. McRae ${ }^{\dagger}$ \\ University of Calgary, Calgary, AB, T2N 1N4 \\ P.M. Danehy: \\ NASA Langley Research Center, Hampton, VA, 23681-2199 \\ E. Gallo ${ }^{\S}$ L. Cantu ${ }^{* *}$, G. Magnotti ${ }^{\dagger \dagger}$, A. Cutler ${ }^{\dagger}$ \\ The George Washington University, Newport News, VA, 23602 \\ R.D. Rockwell ${ }^{\S \S}$, C.P. Goyne ${ }^{* * *}$, J.C. McDaniel ${ }^{\dagger \dagger \dagger}$ \\ University of Virginia, Charlottesville, VA, 22904
}

\begin{abstract}
Hydroxyl radical $(\mathrm{OH})$ planar laser-induced fluorescence (PLIF) measurements were performed in the University of Virginia's dual-mode scramjet experiment. The test section was set up in configuration $A$, which includes a Mach 2 nozzle, combustor, and extender section. Hydrogen fuel was injected through an unswept compression ramp at two different equivalence ratios. Through the translation of the optical system and the use of two separate camera views, the entire optical range of the combustor was accessed. Single-shot, average, and standard deviation images of the OH PLIF signal are presented at several streamwise locations. The results show the development of a highly turbulent flame structure and provide an experimental database to be used for numerical model assessment.
\end{abstract}

\section{Introduction}

$T^{1}$ HE main motivators for developing scramjet engines are to realize high speed, long distance transport, improve missile efficiency, and to reduce the cost of placing payloads into orbit. The inherent advantage of scramjets over conventional rocket engines is the ability to use atmospheric oxygen instead of carrying an onboard oxidizer. This results in an increase in the engine's specific impulse over a wide range of Mach (Ma) numbers. ${ }^{1}$ A recent analytical study by Tetlow and Doolan shows that, for a given payload mass, a hydrogen- or hydrocarbon-fueled scramjet stage uses significantly less fuel than a fully rocket-powered stage for orbital insertion. ${ }^{2}$ They also show, however, that to be economically viable, the scramjet stage must be reusable since it has more structural mass than an equivalent fully rocket-powered system. This requires a robust design, a long operating life, and an easily recoverable system. A logical solution is to integrate the engine with a lifting vehicle airframe.

Although recent flight experiments ${ }^{3,4}$ and demonstration projects ${ }^{5,6}$ have shown that scramjets are feasible in principle, many technical hurdles still remain. One significant hurdle is that the engine is unable to operate over the complete range of flight conditions $(0<\mathrm{Ma}<25)$ needed to achieve orbital insertion. ${ }^{7}$ Scramjets rely on aerodynamic compression through a series of oblique shock waves and can only operate over a limited Mach number range $(5<\mathrm{Ma}<15){ }^{8,9}$ Through normal-shock compression and combustion at subsonic speeds, ramjets

\footnotetext{
* Assistant Professor, Department of Mechanical and Manufacturing Engineering, Member AIAA.

${ }^{\dagger}$ Graduate Student, Department of Mechanical and Manufacturing Engineering, Member AIAA.

$\$$ Research Scientist, Advanced Sensing and Optical Measurement Branch, MS 493, Associate Fellow AIAA.

$\S$ Doctoral Student, Department of Mechanical and Aerospace Engineering, Member AIAA.

** Doctoral Student, Department of Mechanical and Aerospace Engineering, Member AIAA.

${ }^{i \dagger}$ Doctoral Student, Department of Mechanical and Aerospace Engineering, Member AIAA.

\$ Professor, Department of Mechanical and Aerospace Engineering, Associate Fellow AIAA.

$\S$ Senior Scientist, Department of Mechanical and Aerospace Engineering, Member AIAA.

${ }^{* * *}$ Research Associate Professor, Department of Mechanical and Aerospace Engineering, Associate Fellow AIAA.

${ }^{i t \dagger}$ Professor, Department of Mechanical and Aerospace Engineering, Associate Fellow AIAA.
} 
provide thrust at high specific impulses and over lower Mach number ranges (e.g. $3<\mathrm{Ma}<6$ for a hydrogen-fueled ramjet). ${ }^{10}$ Similar to scramjets, ramjets have few to no moving parts, resulting in a relatively simple design for manufacture and reliability.

The integration of a ramjet and a scramjet into a single engine is referred to as a dual-mode scramjet. In this type of engine both subsonic and supersonic modes of combustion are possible, which extends the total operating Mach number range. With improvements in computer processing power, it is becoming more feasible to design and optimize these engines using computational fluid dynamics (CFD). Unfortunately, the flowfield associated with a dual-mode scramjet engine is difficult to predict. The flow includes non-linear interactions between turbulence and combustion, dynamic movement of shock waves, mixed regions of subsonic/supersonic flow, flow separation, fuelair mixing, transition to turbulence, chemistry, and real gas effects. Even in the scramjet mode, there are regions of subsonic flow near the wall and the injector that interact with supersonic flow in the core. ${ }^{10}$ In addition, the engine geometry, including the isolator length, plays a large role in determining the type of flow that enters the combustor. ${ }^{7}$ Only through experimental validation can numerical modeling be trusted to accurately predict this type of flow.

Model validation is a significant objective of the University of Virginia's (UVa) continuous flow, dual-mode Scramjet Combustion Facility (UVaSCF). This facility operates as part of the National Center for Hypersonic Combined-Cycle Propulsion (NCHCCP) program, funded by the Air Force Office of Scientific Research (AFOSR) and NASA. The UVaSCF accommodates several flow configurations and can replicate conditions representative of both ramjet and scramjet modes of combustion. Using a series of non-intrusive diagnostics, large amounts of both qualitative and quantitative information can be acquired without perturbing the flow. Through an overlap in the types of data extracted from the different techniques, there is redundancy in the data set, which is important for credibility. The redundancy also offers information needed to determine the limits of some of the techniques. Measurements using focused schlieren, ${ }^{11}$ stereoscopic particle image velocimetry (SPIV) ${ }_{11}^{11}$ tunable diode laser absorption spectroscopy (TDLAS), ${ }^{12}$ tunable diode laser absorption tomography (TDLAT), ${ }^{13}$ coherent anti-Stokes Raman spectroscopy (CARS), ${ }^{14}$ and hydroxyl radical $(\mathrm{OH})$ planar laser induced fluorescence (PLIF) ${ }^{15}$ are in the process of being performed in this facility.

The OH PLIF measurements in the combustor section of configuration A, as described below, are reported herein. The two primary objectives are to produce a high quality experimental dataset for model assessment and to learn more about the physics of the flow through the interpretation of the PLIF images. The position of the PLIF measurement plane and the equivalence ratio were varied for completeness and to provide more than one condition for comparison.

\section{UVa Dual-Mode Scramjet Facility}

The UVaSCF is a vertically oriented, continuous running, direct-connect scramjet facility. The modular nature of the facility allows it to be assembled in various configurations, four of which are shown in Fig. 1. In the current study, configuration A was selected. A schematic of the facility, shown in Fig. 2, displays a large electrically heated air supply, test section, and exhaust system. Because the exhaust system is not connected directly to the test section, exhaust is vented from the extender to atmospheric pressure. The electrical resistance heater ensures that no vitiated gas or other contaminants enter the freestream flow before entering the test section. The stagnation pressure and temperature are roughly $300 \mathrm{kPa}$ and $1200 \mathrm{~K}$, respectively. 

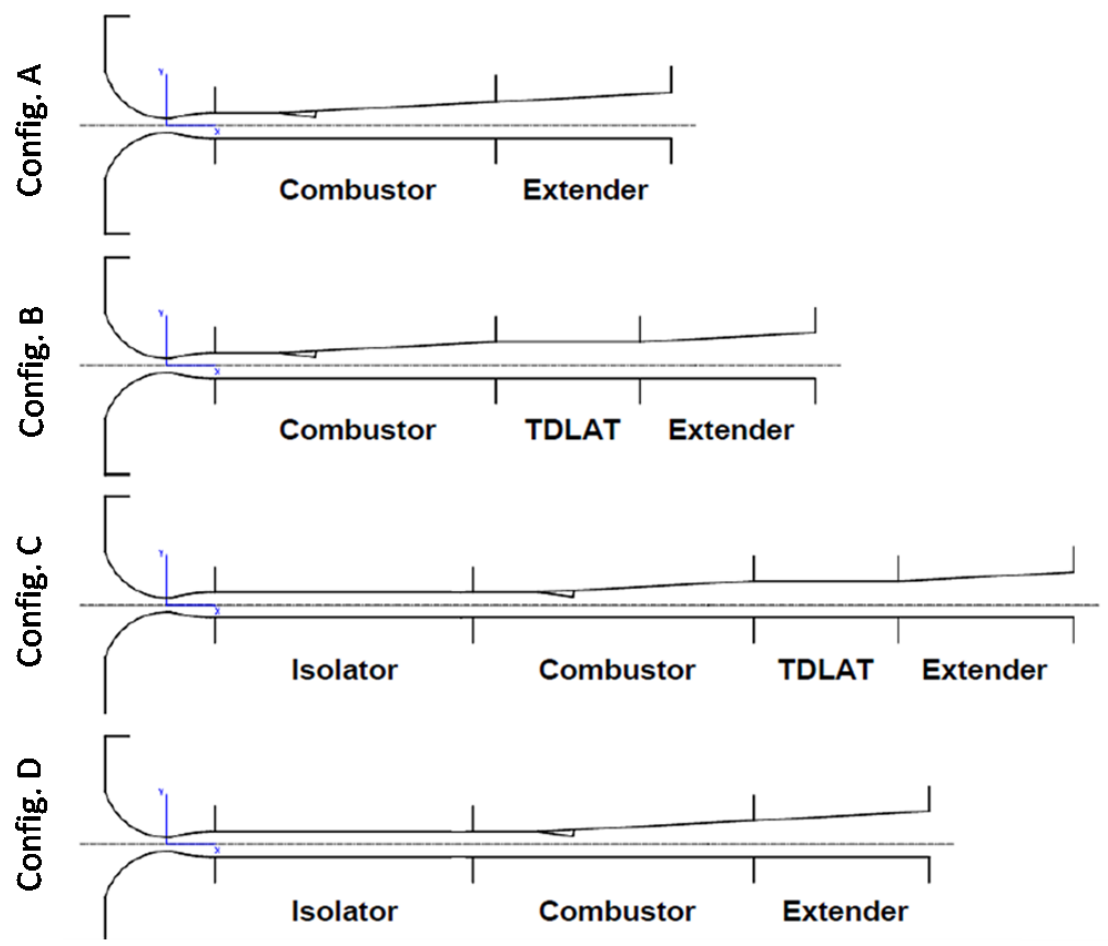

Figure 1: Test section configurations. Configuration $A$ is used in current study.

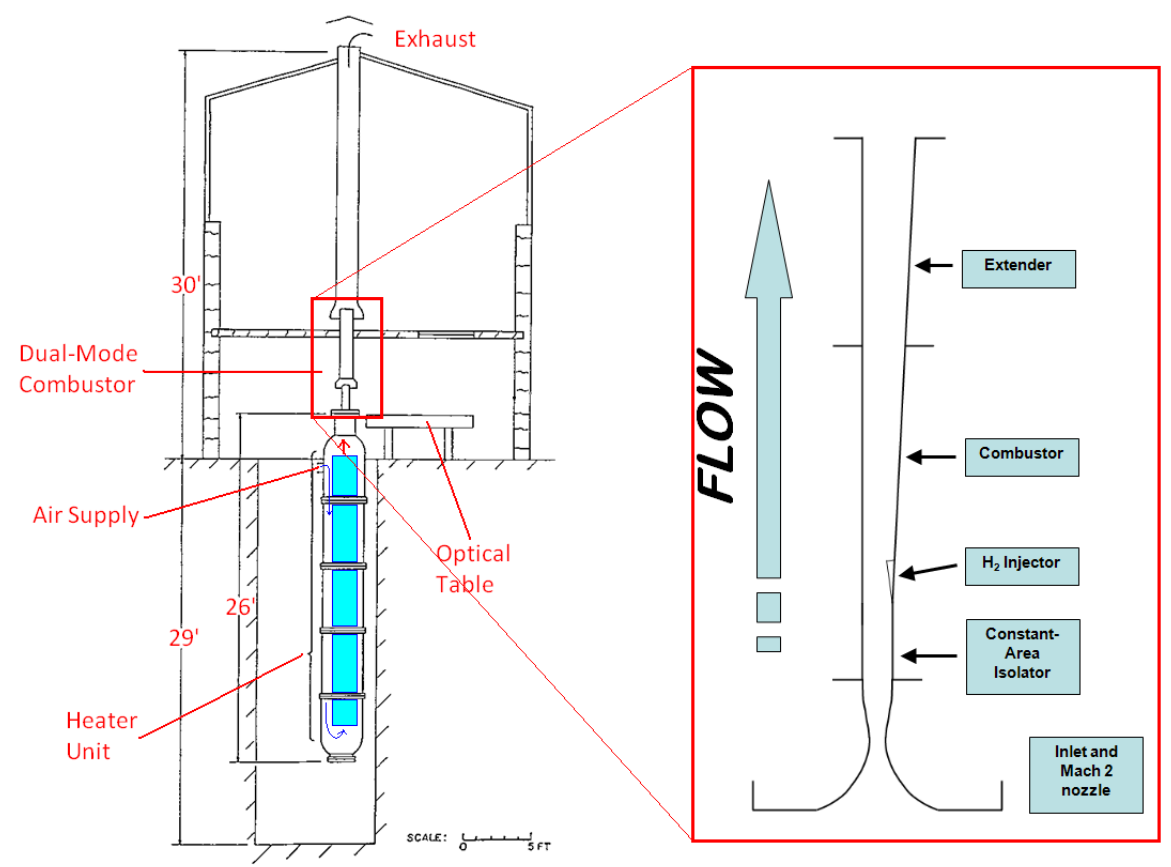

Figure 2: UVaSCF facility. Configuration D displayed as shown in Fig. 1. Reproduced from Ref [15].

A Mach 2 nozzle is used to accelerate the flow through the test section while enthalpy conditions correspond to flight at Mach 5. ${ }^{14}$ The complete test section (only a subset of which is used herein) includes four modules: an isolator, a combustor, a TDLAT section, and an extender. Figure 3 shows a three dimensional CAD rendering of each of the modules and the locations of the various diagnostic locations. Note that the test section has been 
reoriented horizontally in this figure. A constant area isolator section allows a shock train to stabilize ahead of the combustor section. The module has optical access to perform SPIV, CARS, and TDLAS measurements. The combustor section includes a single, unswept, wall-mounted compression ramp with a fuel injector. The compression ramp is characterized by a width of $W=12.7 \mathrm{~mm}$, height of $H=6.35 \mathrm{~mm}$, and the ramp surface is oriented at $10^{\circ}$ relative to the divergent wall of the combustor. The injector is positioned on the downstream side of the ramp and includes a converging-diverging conical nozzle to accelerate the hydrogen fuel to Mach 1.7. ${ }^{16}$ By controlling the mass flow rate of the fuel, the equivalence ratio $(\phi)$ was varied in the experiments from 0.17 to 0.34 , with most of the data obtained at these two extremes. The equivalence ratio is defined as the mixture fuel-to-oxidizer ratio normalized by the stoichiometric fuel-to-oxidizer ratio. The centerline fuel jet direction is oriented approximately $7.1^{\circ}$ relative to the freestream flow. The combustor wall diverges from the opposing wall at an angle of $2.9^{\circ}$ starting from the leading edge of the compression ramp. To allow for TDLAT measurements, a constant area section is connected to the exit of the combustor. Finally, an expanding extender section completes the test section with a divergence angle of $2.9^{\circ}$.

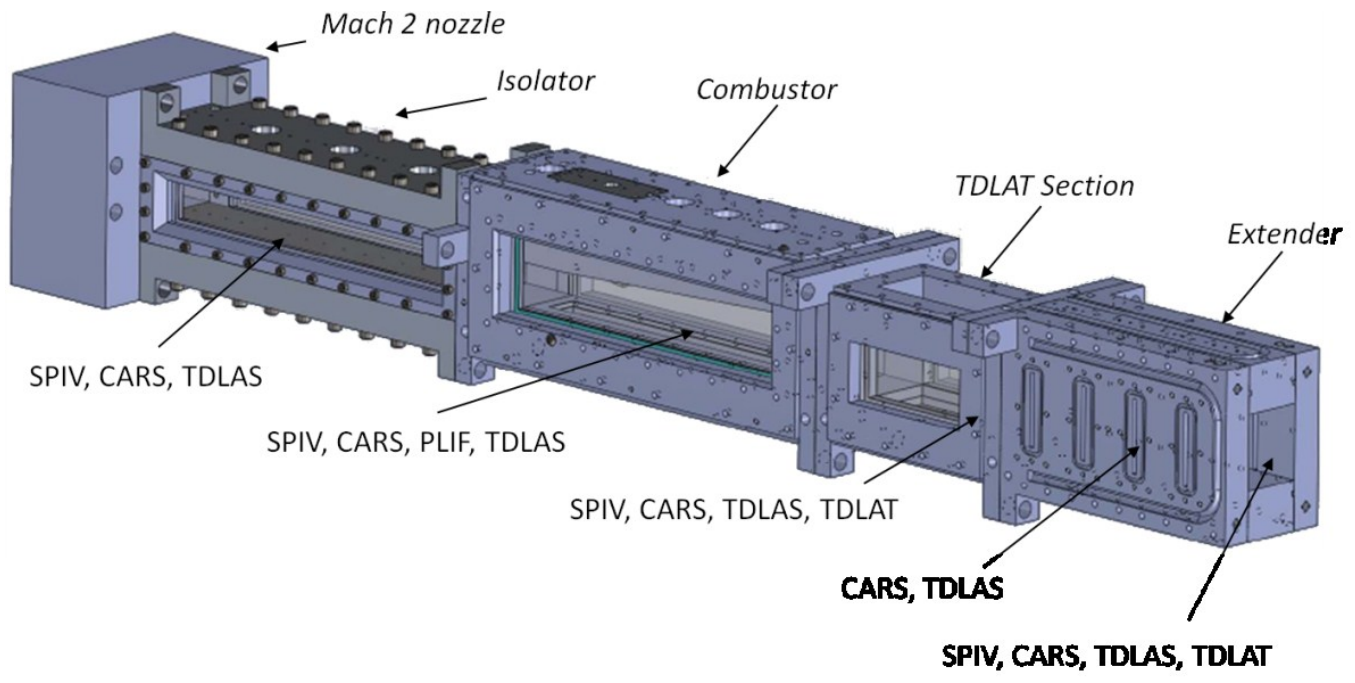

Figure 3: Available test section modules and diagnostic locations. Configuration $\mathrm{C}$ displayed as described in Fig. 1. OH PLIF measurements in combustor section of configuration A are reported in current study.

Table 1 lists each diagnostic technique and describes the quantity that is measured. Note that for the current study, only the PLIF measurements in the combustor section are reported.

\section{Table 1: Summary of diagnostic techniques}

\begin{tabular}{|l|l|}
\hline Technique & Measured Quantities \\
\hline SPIV & 3D velocity on 2D plane \\
\hline TDLAS & Path averaged temperature and species concentration $\left(\mathrm{H}_{2} \mathrm{O}\right)$ \\
\hline TDLAT & Spatial distribution of temperature and species concentration $\left(\mathrm{H}_{2} \mathrm{O}\right)$ \\
\hline PLIF & Planar OH distribution (qualitative) \\
\hline CARS & Point measurement of temperature and species concentration $\left(\mathrm{N}_{2}, \mathrm{O}_{2}, \mathrm{H}_{2}\right)$ \\
\hline
\end{tabular}




\section{PLIF System Setup}

To facilitate OH PLIF in the combustor section, ultraviolet (UV) grade fused silica windows were placed along the side walls. This allowed for the transmission of the UV laser light from the laser source needed to excite the $\mathrm{OH}$ molecules and transmission of the subsequent fluorescence to the camera. An injection seeded, pulsed Nd:YAG was frequency doubled to $532 \mathrm{~nm}$ to pump a Spectra Physics PDL-2 dye laser, outputting tunable light at $567.1 \mathrm{~nm}$. The output of the dye laser was frequency doubled using a component of a wavelength extender (WEX) module to produce UV light at $283.55 \mathrm{~nm}$. A HighFinesse WS-6 wavemeter was used to monitor the laser wavelength. At this wavelength the $\mathrm{Q}_{1}(8)$ transition of $\mathrm{OH}$ was excited. This transition was selected because the PLIF signal is relatively insensitive to temperature, allowing the signal to be representative of $\mathrm{OH}$ concentration. ${ }^{17} \mathrm{O}$ 'Byrne et al. probed this transition to perform OH PLIF measurements in a supersonic combustion using cavity injection in the T3 free-piston shock tunnel. ${ }^{17}$ The Nd:YAG and the dye laser were located on a mobile cart originally used as part of a CARS setup,$^{14}$ which was later modified to include the doubling crystal from a WEX unit and associated beam separation and relay optics. Note that the laser operated at $20 \mathrm{~Hz}$ but the CCD cameras framed at $10 \mathrm{~Hz}$, as the PI-MAX2 camera could not acquire full resolution images at greater than $13 \mathrm{~Hz}$.

As shown in Fig. 4 (left), sheet forming optics, a dye cell, and a CoolSnap sheet-profiling camera were mounted on a motorized vertical translation stage that was secured to a large optical table adjacent to the test section. A series of UV mirrors relayed the beam from the mobile laser cart onto the translation stage. The beam was expanded in one direction with a UV cylindrical lens $(\mathrm{f}=25 \mathrm{~mm})$ and then collimated with a UV spherical lens $(50 \mathrm{~mm}$ diameter, $\mathrm{f}=250 \mathrm{~mm}$ ). This produced a $50 \mathrm{~mm}$ wide and less than $1 \mathrm{~mm}$ thick laser sheet that was directed into the combustor, perpendicular to the flow axis. A beam splitter was placed between the test section and spherical lens to redirect part of the sheet up towards a Rhodamine dye filled quartz cell. Synchronized to the laser pulse, the unintensified CoolSnap camera imaged the dye cell to measure any spatial non-uniformity in the laser sheet on a shot-by-shot basis. Spatial non-uniformity affects the PLIF signal since fluorescence is proportional to the excitation energy in the linear regime. The sheet profiling information was later used to correct the PLIF images.

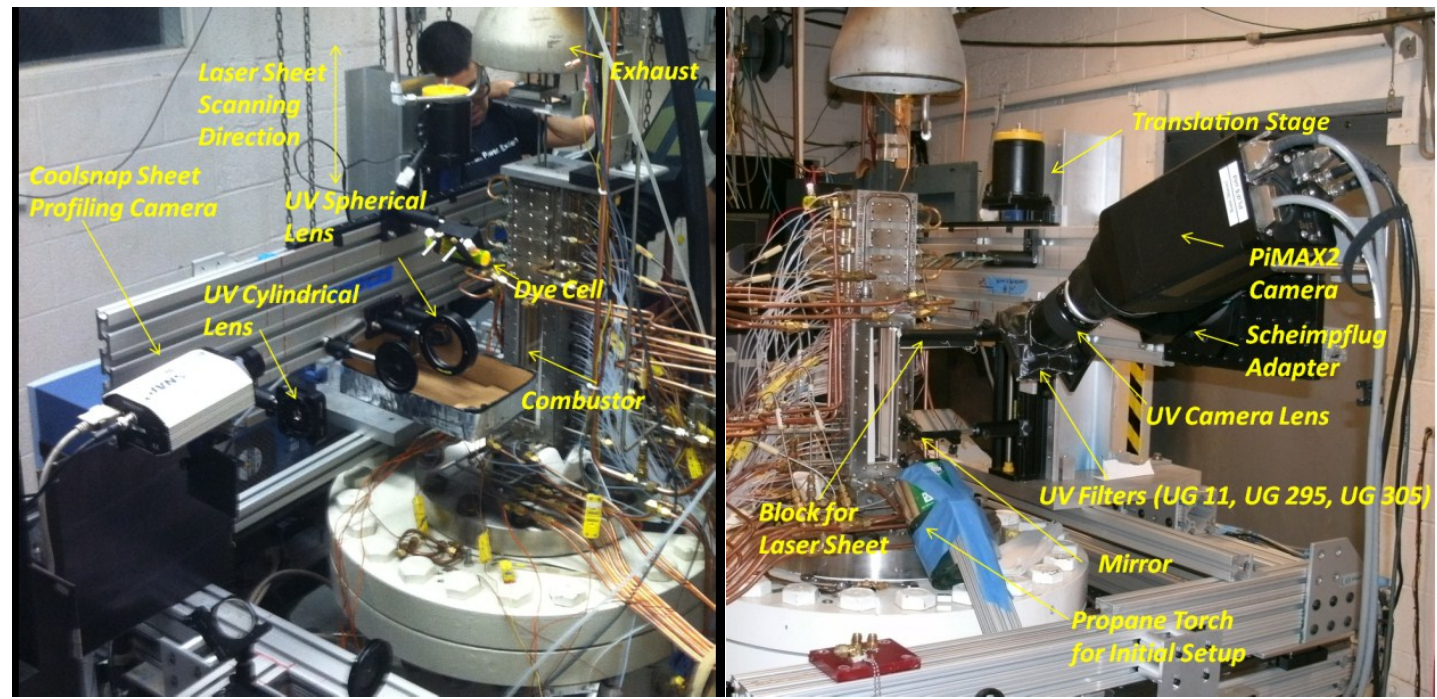

Figure 4: Sheet forming optics and sheet profiling camera (left); and ICCD camera (right).

The right image in Fig. 4 shows a PI-MAX2 ICCD (intensified charge-coupled device) camera with a UV Nikkor F/4.5 $105 \mathrm{~mm}$ focal length lens that was used to image the PLIF signal. A beam block was placed at the laser sheet level to protect the camera. Schott UG11 $(2 \mathrm{~mm})$, WG295 $(2 \mathrm{~mm}+1 \mathrm{~mm})$, and WG305 $(2 \mathrm{~mm})$ filters were used to reduce laser scatter off the facility windows and combustor wall surfaces. A propane torch was used as a source of $\mathrm{OH}$ for initial calibration to optimize the laser's wavelength and help focus the PI-MAX2 camera. In addition to a streamwise traverse of the equipment, the laser sheet, mirrors and camera could translate approximately 10 inches in the horizontal direction, allowing the propane torch, dotcards, and imaging cards to be placed in the measurement plane without re-alignment of the equipment. A mirror was placed near the combustor window to allow the camera to image a more perpendicular view of the sheet than would have been otherwise possible with a direct camera view. A Scheimpflug adapter was used to help focus the camera on the image plane. Translating the sheet-forming optics, mirrors, and cameras along the flow axis allowed for a range of streamwise positions in the flow to be investigated. 
The analysis of OH PLIF results herein are non-dimensionalized relative to the compression ramp height, $H$, with an emphasis on measurement planes at $x / H=6,12$, and 18 downstream of the injector plane. These measurement planes coincide with CARS measurements also taken with this test section configuration. ${ }^{14}$ Unfortunately, geometric constraints near the test section forced two different camera view configurations to be used to access the full range in the streamwise direction (Fig. 5). The first camera view (top) used one mirror and captured images from an upstream position. This view allowed imaging up to the downstream edge of the combustor window $(x / H=23.5)$. However, because the mirror was located near the facility nozzle, the entire system could only be translated up to a position of $x / H=10.2$. In the second camera view (bottom), a pair of mirrors, one of which was positioned further downstream, allowed the camera to image the PLIF signal from a downstream position. This extended the range of images to the upstream edge of the combustor window $(x / H=-3.0)$. With this setup the laser sheet was able to translate upstream of the fuel injector. Because the camera imaged the PLIF signal from the opposite side of the laser sheet relative to camera view 1, the additional reflection from the second mirror was naturally corrected. As such, the PLIF in both views was captured from a perspective as though looking upstream with the laser-sheet coming in from the right. The images were later corrected in post-processing to match results from previous studies, ${ }^{14,15}$ such that the laser sheet would enter from the left. Another useful feature of this setup was that two of the three mirrors could be installed in advance of the experiment. Half-way through the experiment, one mirror was removed and another installed quickly and with minimal alignment to change the configuration from camera view 1 to 2 .

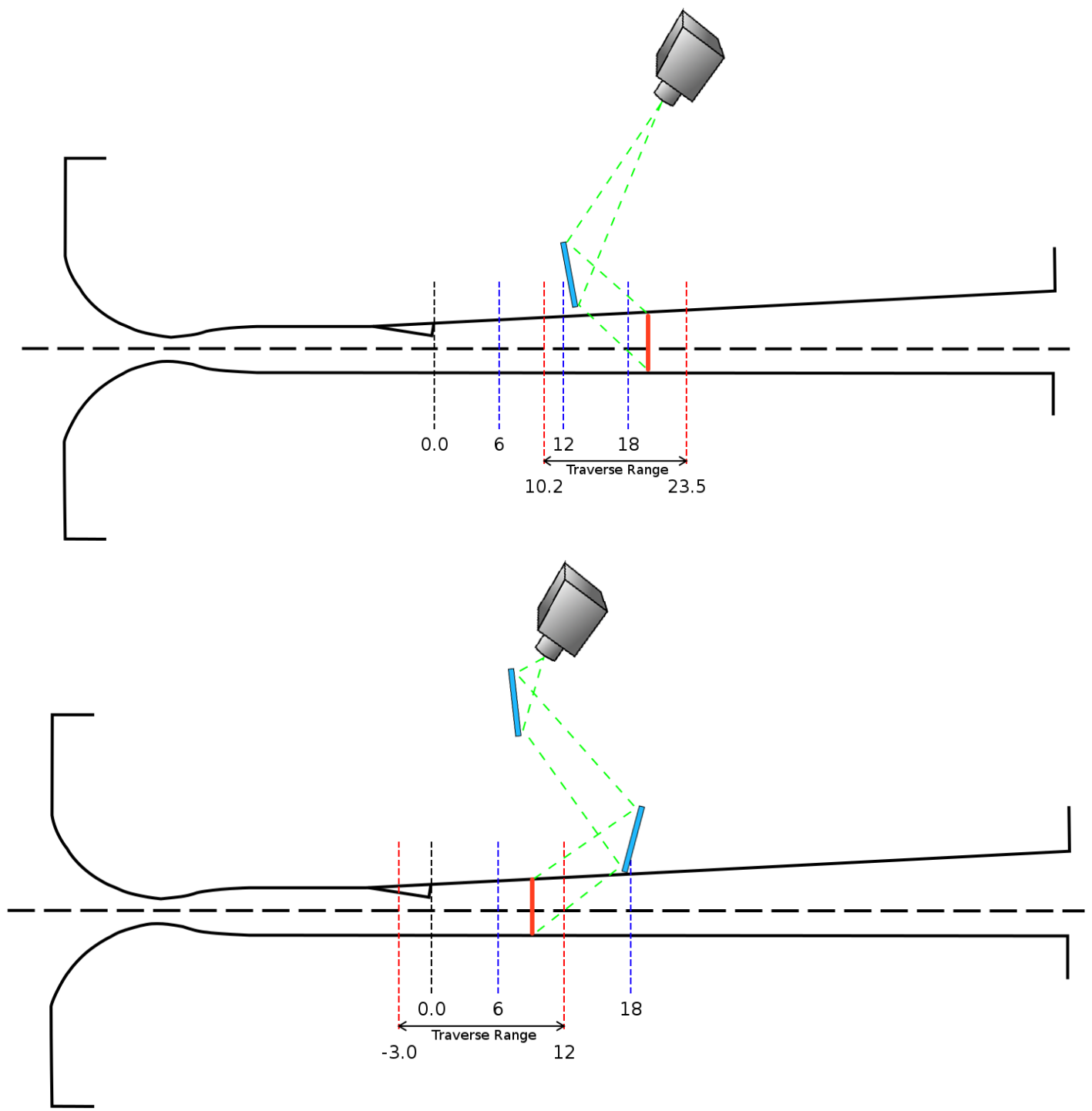

Figure 5: Camera view 1 (top) and camera view 2 (bottom). Dimensions are displayed in $x / H$ units. 


\section{Post Processing}

Optical distortions and distortions due to the camera perspective were corrected through post processing of the PLIF data. The process loosely follows the procedure outlined by Danehy et al. for nitric oxide (NO) PLIF. ${ }^{18}$ The post processing involved five major steps: dotcard analysis, unwarping of PLIF data, calibration sheet analysis, creation of correction sheets, and applying final corrections. The dotcard is a rectangular grid of uniform square dots with 0.125 -inch spacing printed on white card stock. Given the computer generated template dotcard and the experimentally captured distorted dotcard images, the optical distortion was corrected using a plug-in for ImageJ, ${ }^{19}$ a freeware image processing software developed at the National Institutes of Health. The plug-in, a modification of Unwarp $^{20}$ for working with image stacks, was used to apply the unwarping algorithm across each set of PLIF data to correct for the camera distortions. Note that the terminology "unwarp" is used herein to describe the process of using UnwarpJ to correct PLIF image distortions due to perspective and lens distortions.

As described previously, two separate camera views were used to access the full streamwise range in the combustor, which was only limited by the length of the windows. Images of the dotcards in the plane of the laser sheet are shown for both camera views in Fig. 6. In camera view 1, the angle of the Scheimpflug adapter was adjusted to optimize the picture quality. The Scheimpflug adapter was not adjusted after the switch between camera views because of time constraints in using the facility. In addition, camera view 2 had more perspective distortion, resulting in a lower $y$-pixel magnification. As a result, lower quality images were acquired using the camera view 2 configuration compared to images from camera view 1.
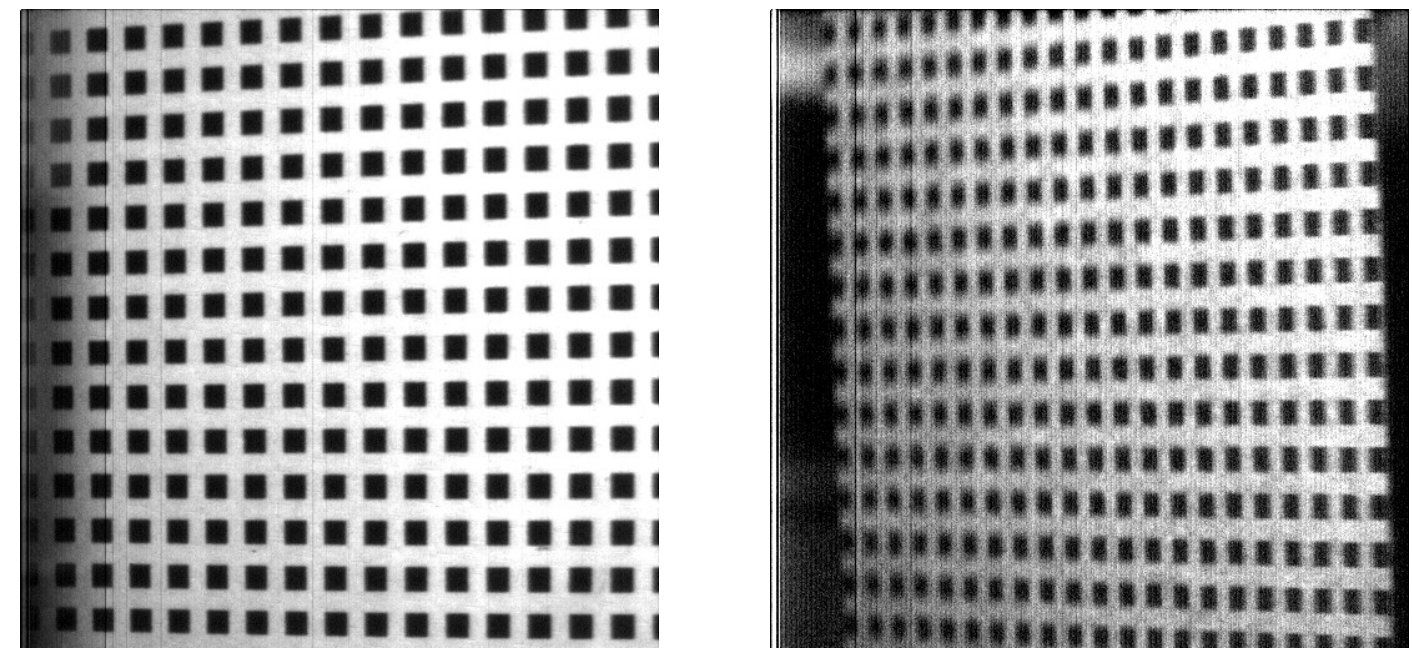

Figure 6: Dotcards captured in the test section for camera view 1 (left) and 2 (right).

Figure 7 shows a sample raw PLIF image (left) and 2000-shot average (right) for a representative run. These images are unprocessed and were acquired using camera view 1 at a streamwise distance of $x / H=12$ and at a fuel equivalence ratio of $\phi=0.17$. The laser sheet enters the test section from the left in the positive $z$-direction in the images presented, as though looking downstream from the given $x / H$ plane. Fuel is injected in the center of the duct in the cross-stream direction and upstream of the image plane. The positive $y$-direction is towards the injector wall. Combustion occurs in semi-circular shaped regions of mixed fuel and air. Turbulence wrinkles the flame surface, as visible in the single-shot images. With a 2000-shot average, however, the $\mathrm{OH}$ distribution appears smooth. The lines left and right of the PLIF are scatter off of the test section windows and the above-mentioned filters were added to reduce this scatter; however, this scatter signal still exceeds the PLIF signal by as much as a factor of 15 . Further analysis of the fully corrected images is discussed in the Results section. 


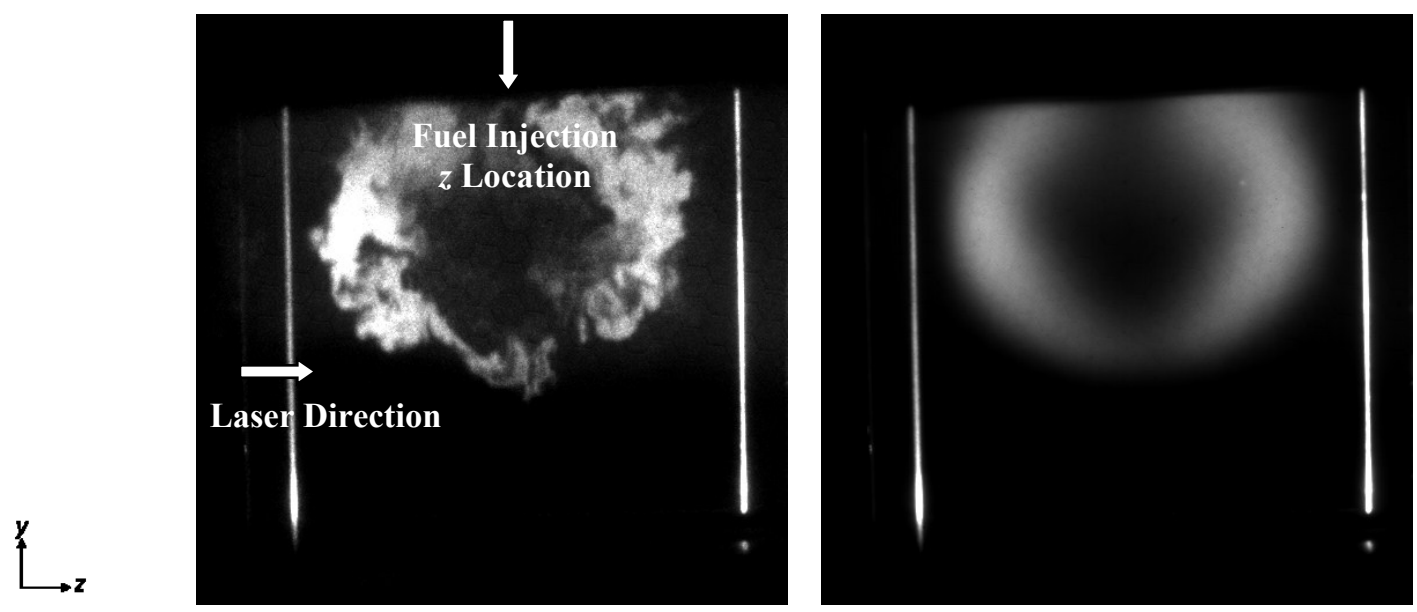

Figure 7: Unprocessed single shot (left) and 2000-shot average (right) PLIF images; $\phi=0.17, x / H=12$.

To illustrate the unwarping process, Fig. 8 shows both a raw (left) and an unwarped (right) 2000-shot average PLIF image corresponding to camera view 1. Based on the laser scatter from the windows, shown in the right image of Fig. 8 , it was apparent that even after the unwarping process a small rotation of 0.7 degrees $(\mathrm{CCW})$ needed to be applied to the dataset. It is likely that the dotcard was slightly rotated with respect to the facility test section, causing the rotation error. Similarly, there was slight divergence between the two lines of laser scatter corresponding to each window surface. This indicates either a positioning error of the dotcard or a divergence between the combustor side walls in the facility. Note that the divergence is less than 0.3 degrees and does not affect the overall interpretation of the results. In cases where the camera and optics were traversed downstream, there was a small shift of the PLIF signal and laser scatter of approximately 5 pixels over the entire scan range of camera view 1 . This occurred because of a slight misalignment of the translation stage with respect to the facility. This shift was corrected in the final images.
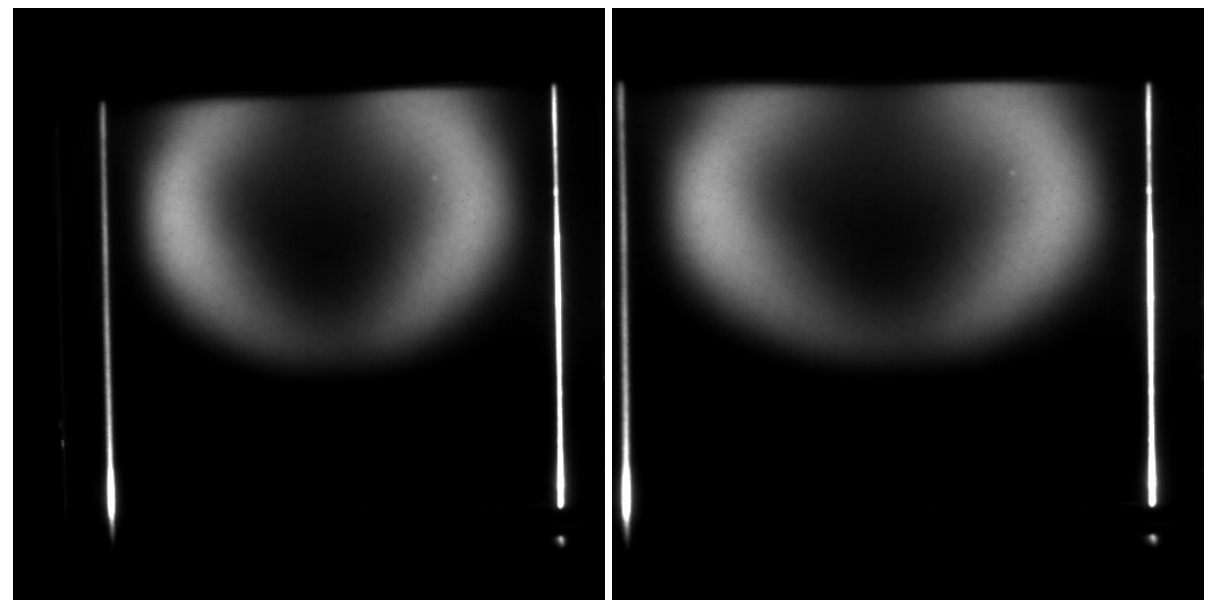

Figure 8: Raw (left) and unwarped (right) 2000-shot average PLIF images; Camera view 1, $\phi=0.17, x / H=$ 12.

Figure 9 shows the unwarping process for camera view 2, with raw (left), raw with crop and stretch (center), and unwarped (right) 2000-shot averages. As the dotcard image from camera view 2 contains unusable data beyond the edges of the cardstock (see Fig. 6, right image), a smaller region on the dotcard, which corresponds to the PLIF signal, was selected and cropped. Both the cropped dotcard and PLIF images from camera view 2 were then stretched to match the spatial resolution used for camera view 1. Note that stretching does introduce some interpolation errors into the camera view 2 images. Prior to stretching, the spatial resolution in the $y$ and $z$ directions for camera view 2 were 11.97 pixels $/ \mathrm{mm}$ and 6.87 pixels $/ \mathrm{mm}$, respectively. After stretching and unwarping of camera view 2 , the effective spatial resolution for both camera views was 11.85 pixels $/ \mathrm{mm}$. 

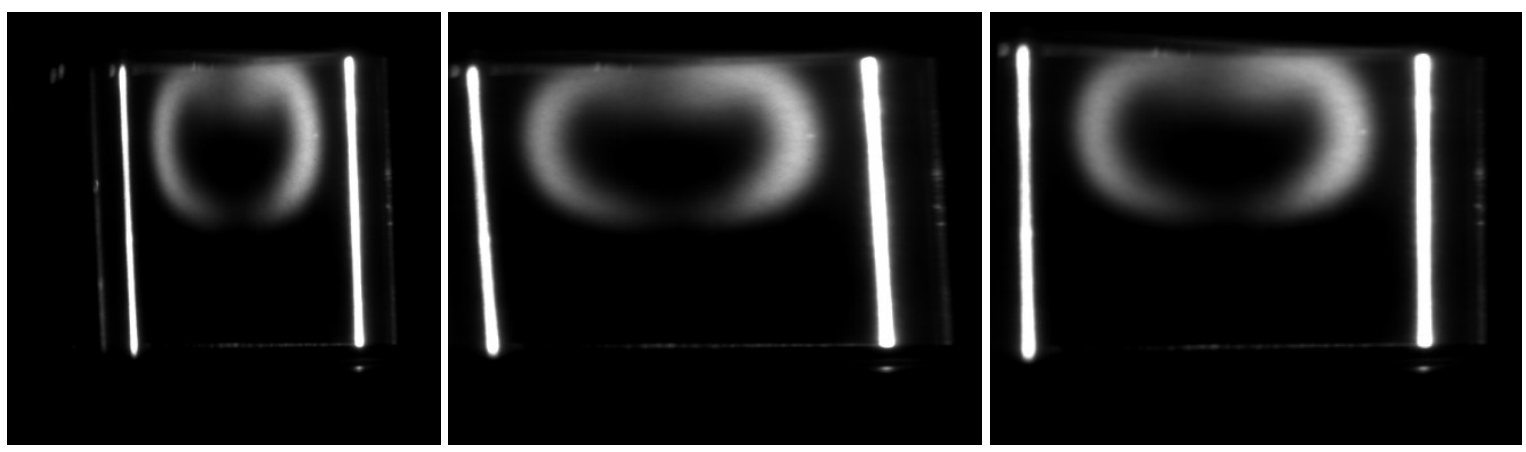

Figure 9: Raw (left), raw with crop and stretch (center), and unwarped (right) 2000-shot average PLIF images; Camera view $2, \phi=0.17, x / H=6$.

After unwarping, the images were corrected for any spatial non-uniformity in the laser sheet using synchronized single-shot images of a Rhodamine dye cell captured from a Photometrics CoolSnap interline CCD camera. To account for differences in the spatial resolution of PI-MAX2 and CoolSnap images, a cardstock with a cutout pattern was placed into the laser sheet path, resulting in a pair of calibration images acquired from both cameras. These calibration images were used to properly align the sheet profiling images to the PLIF images. Figure 10 shows both original and blurred intensity distributions across the dye cell for three separate instances. In addition to high frequency noise, repeatable small scale oscillations are observed in the sheet intensity profiles. These oscillations are likely a result of dust particles on the dye cell or irregularities in the glass material that contains the dye. Application of the unprocessed sheet correction profiles would result in non-physical streaks in the final PLIF data. Therefore, a Gaussian blur with an optimized sigma value of 25 pixels was applied to each sheet profile to eliminate any repeatable oscillations but still retain the overall profile. The blurred sheet profiles were then applied to the PLIF dataset on a shot-by-shot basis. As shown in Fig. 10, this correction is significant, typically changing the signal by a factor of 2 on one side of the image compared to the other. Also, it corrected for variations in laser intensity which could be as much as 50 percent during the test.

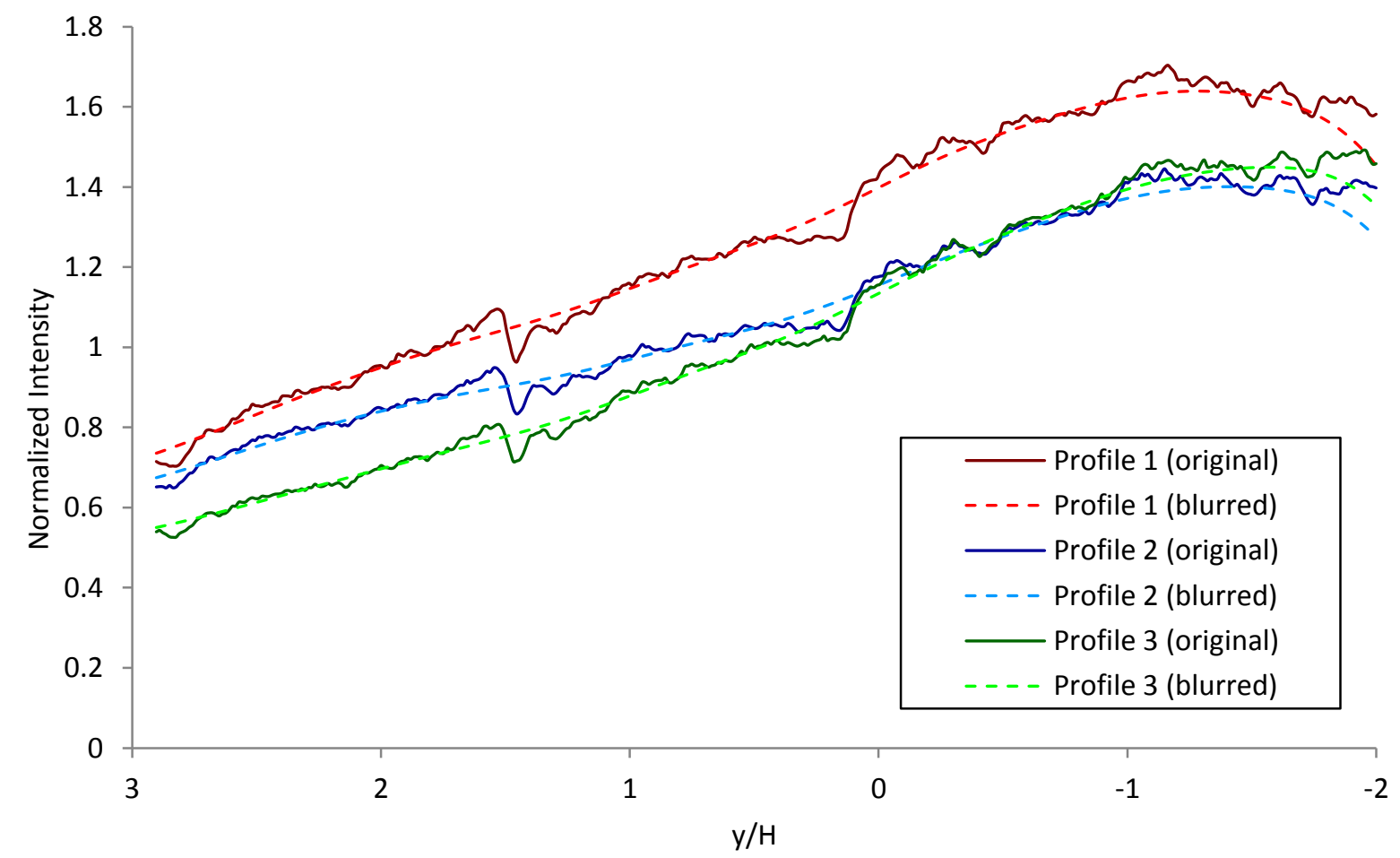

Figure 10: Application of a Gaussian blur to a normalized correction sheet for three arbitrary times; raw correction sheet intensity (solid lines) with corresponding Gaussian blur (sigma $=25)$ overlay (dashed lines). 
Figure 11 shows an unwarped 2000-image average for $\phi=0.17$ and $x / H=12$ before (left) and after (right) laser sheet correction. After the correction, there is a small but noticeable shift in the PLIF signal towards the injector wall. Further post processing (included in both images in Fig. 11) of the PLIF data included background subtraction using an average signal intensity over a region of negligible PLIF signal located near the combustor walls opposite of the injector. The images were then cropped to only include the interior region of the combustor. The laser scatter, however, was included in the images for reference. To account for two separate camera views, the average PLIF intensity from images using camera view 2 were corrected to match the average intensities in overlap regions that occur with camera view 1. A preset Fire color table available in ImageJ was applied to all images with consistent brightness and contrast values.

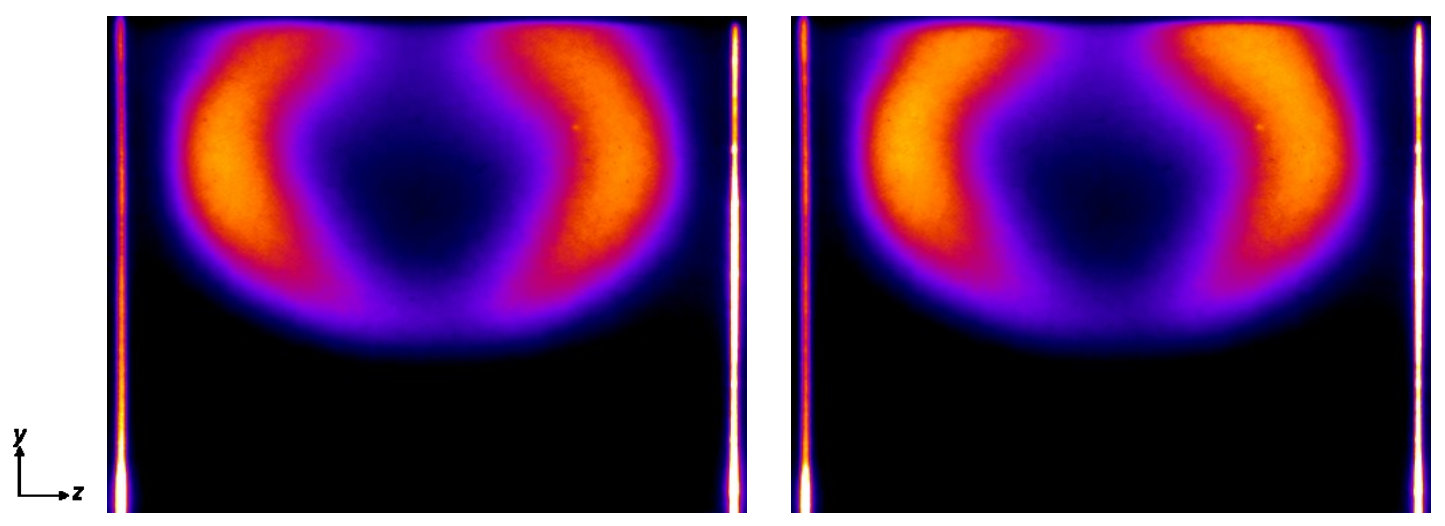

Figure 11: Unwarped 2000-shot average before (left) and after (right) laser sheet correction; Camera view 1, $\phi=0.17, x / H=12$.

\section{Results}

Figure 12 shows a series of single-shot (left), average (center), and standard deviation (right) OH PLIF images for three different streamwise positions downstream of the injector plane. In these images, hydrogen fuel was injected at a global equivalence ratio of $\phi=0.17$. As can be seen from the average PLIF signal images, the overall size of the flame surface is relatively small near the fuel injector but then grows with increasing streamwise distance. In addition to the size, $\mathrm{OH}$ concentration levels also increase. This is a combination of the accumulation of $\mathrm{OH}$ as it convects downstream as well as an increase in the reaction rate as the fuel and air become more mixed. Note that the color table is identical for all of the images in the figure. The single shot images give an indication of the local length scales of turbulence near the reaction zone. The sizes of turbulent structures observed, however, are limited by the camera resolution.

The main source of turbulence production appears to be the shear layer that forms around the compression ramp. The shear layer interacts with the perimeter of the fuel jet, which enhances mixing and increases the rate of heat release. Interestingly, two distinct bands are visible in the standard deviation images. Each band indicates high levels of turbulence and regions where combustion occurs intermittently. The inner band coincides with the fuel-rich region while the outer band coincides with the fuel-lean region. The bands are more distinct near the fuel injector and become less pronounced with increasing streamwise distance. Again, this is likely due to more uniform mixing at planes further downstream. The bands are centered to either side of the peak levels in the mean signal images, as shown in Fig. 13. In this figure, a line plot through the center of the PLIF signal region is shown for the single shot, average, and standard deviation images from Fig. 12 for $x / H=12$. 

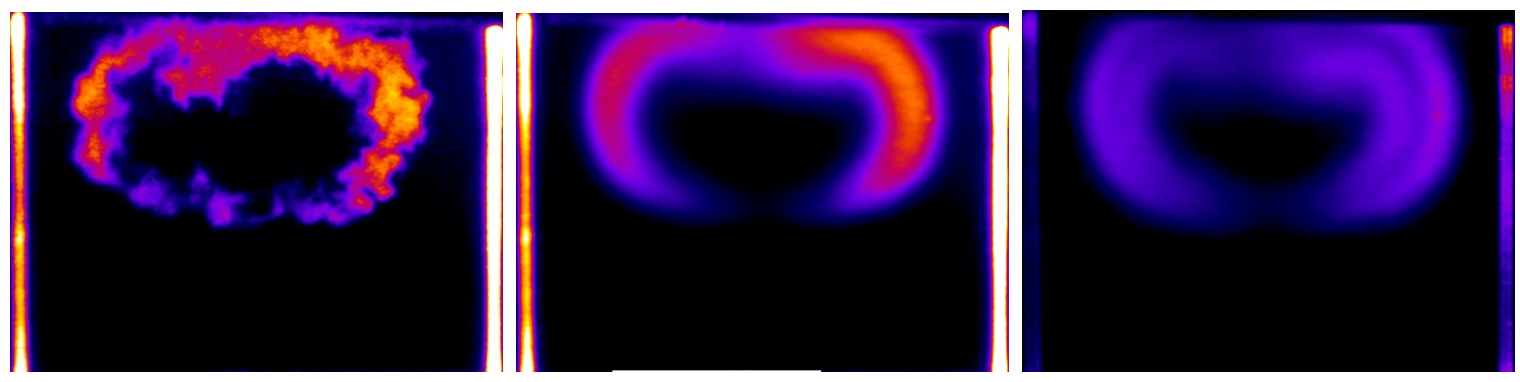

$x / H=6$
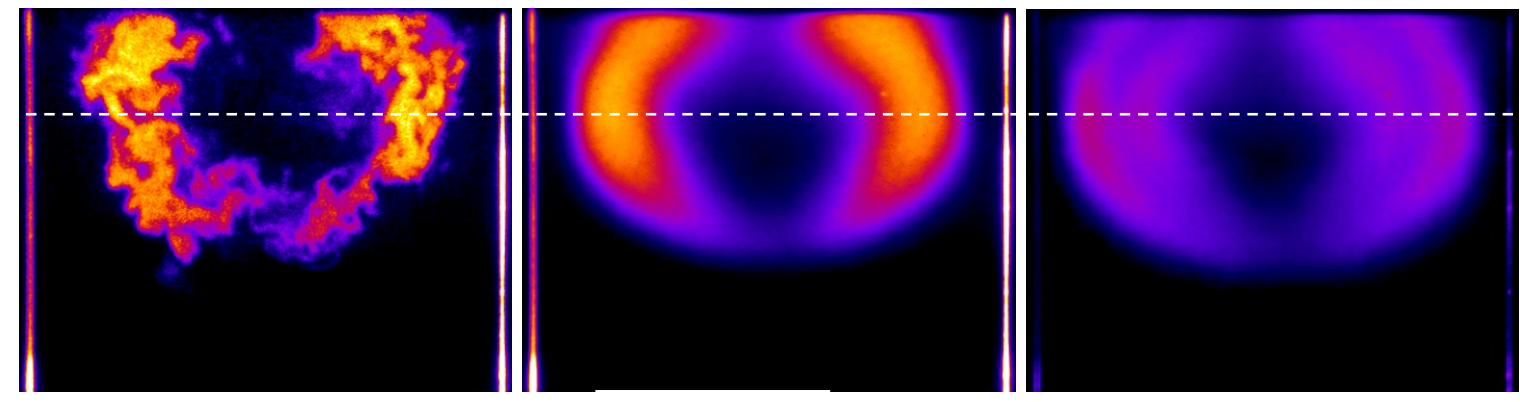

$x / H=12$
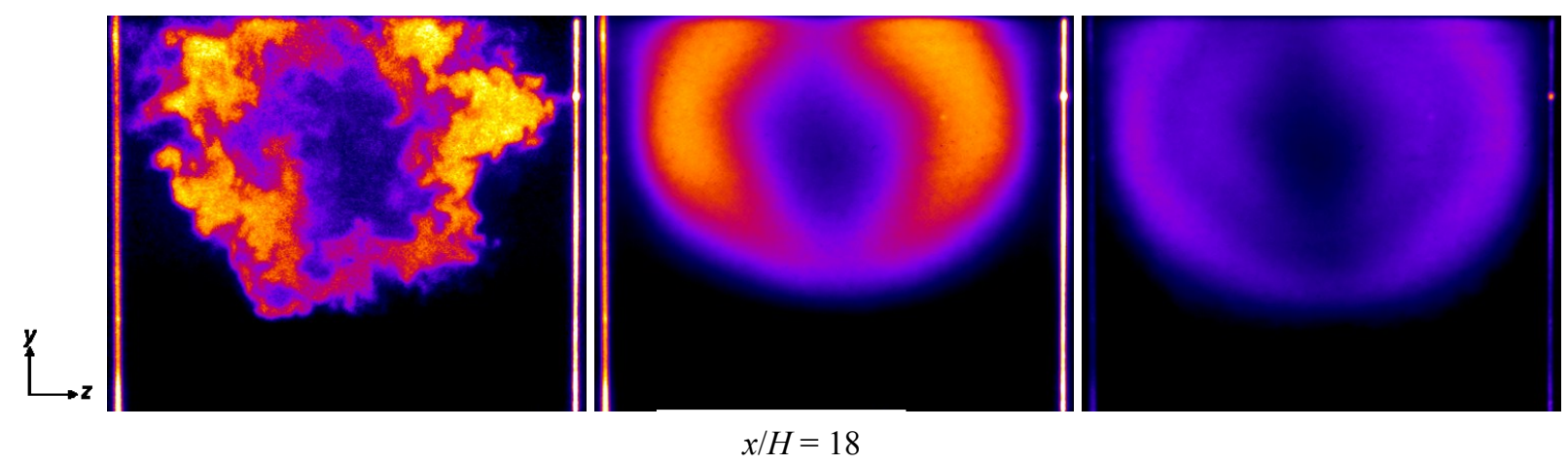

Figure 12: Single-shot (left), average (middle), and standard deviation (right) of the PLIF signal. $\phi=0.17$. Dashed line shows profile location for Fig. 13. 


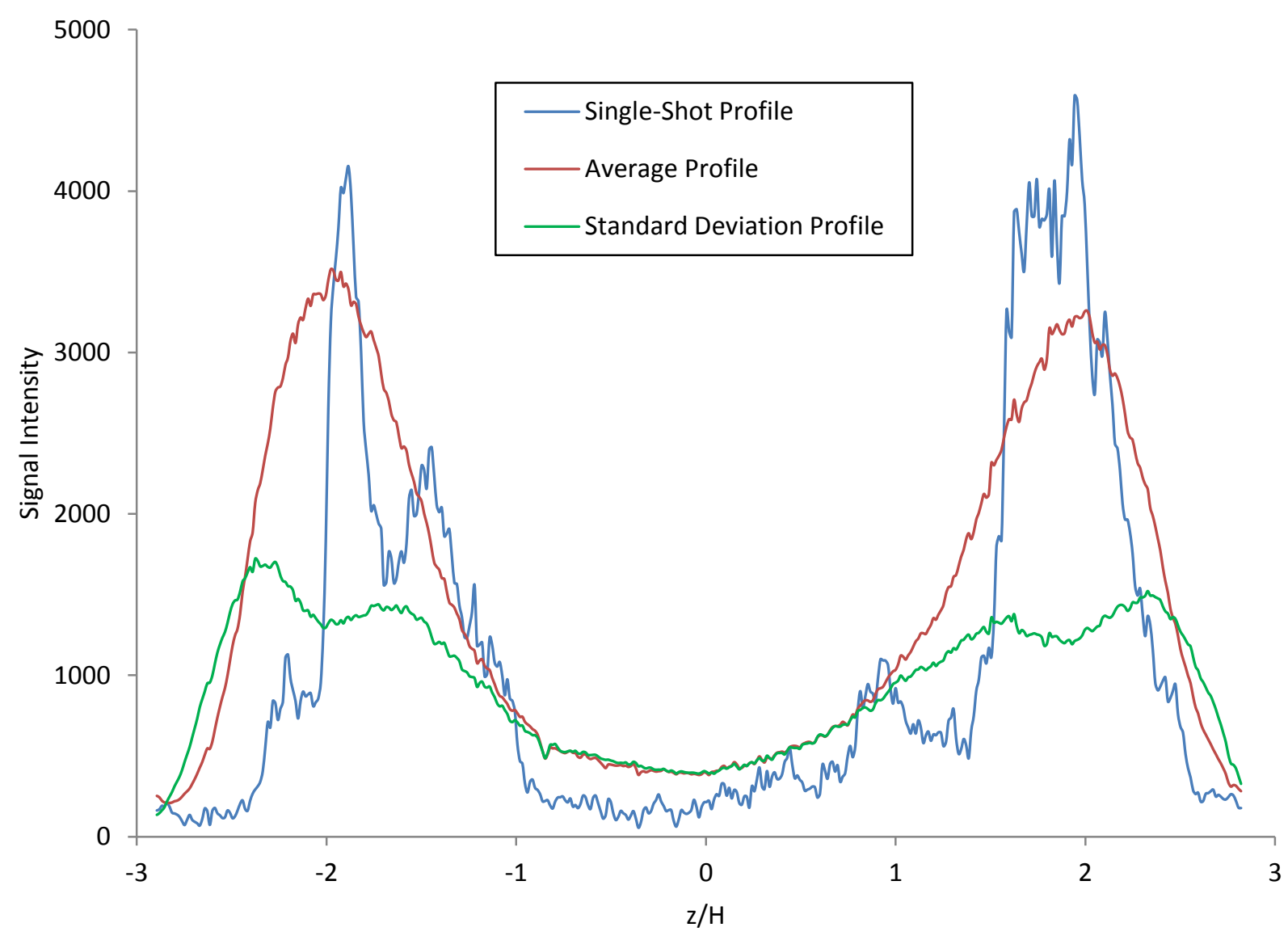

Figure 13: Line plot of $O H$ PLIF signal at an equivalent $y / H$ position for single-shot, average and standard deviation images for $x / H=12$ and $\phi=0.17$.

Figure 14 shows individual single-shot images for an equivalence ratio of $\phi=0.17$ overlaid onto a 3D wireframe of the combustor. During the experiments, scans were performed in the upstream direction towards the injector, from an $x / H$ of 23.5 to 10.2 for camera view 1 , and 12 to -3.0 for camera view 2. Selected $x / H$ planes are shown from which the spreading rate of the jet and three dimensional distribution of the reaction zone can be visualized. Near the injector, combustion only occurs along the channel walls and along the shear layer that forms from the compression ramp sidewalls. The reaction does not initially occur along the jet perimeter near the high-speed core flow. However, at further downstream positions, combustion occurs along all edges of the hydrogen jet. Similarly, Fig. 15 shows a 3D wireframe overlay for the higher equivalence ratio case, $\phi=0.34$, at equivalent $x / H$ positions. Comparing Fig. 14 and Fig. 15, fluorescence is evidently greater for the higher equivalence ratio; however, there is greater distribution of $\mathrm{OH}$ at the lower equivalence ratio for downstream planes nearer the injector. This suggests that combustion is uniformly distributed more rapidly for lower equivalence ratios. 


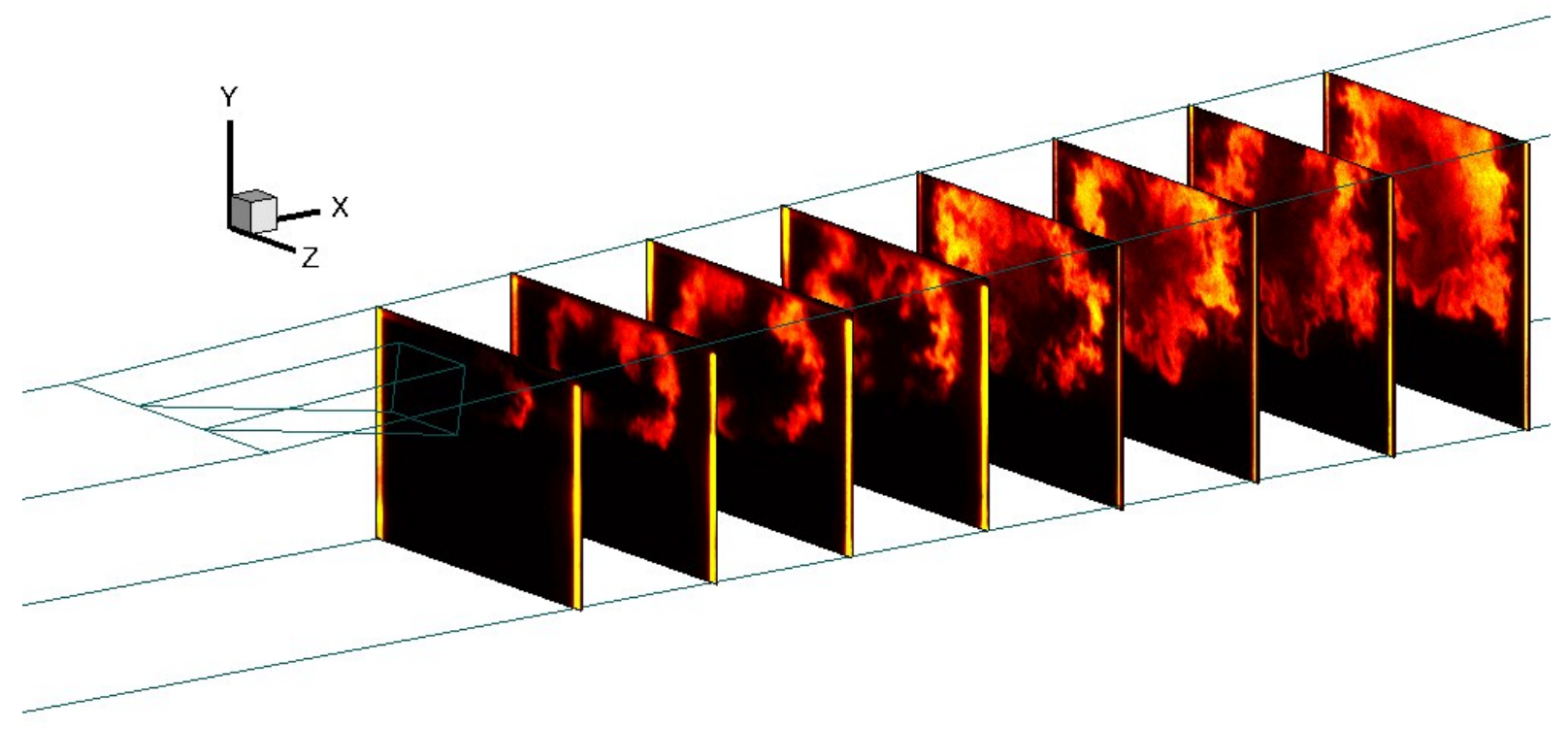

Figure 14: Streamwise distribution of PLIF overlaid onto wireframe drawing of combustor. $\phi=0.17$, scan from $x / H=22$ to 1 .

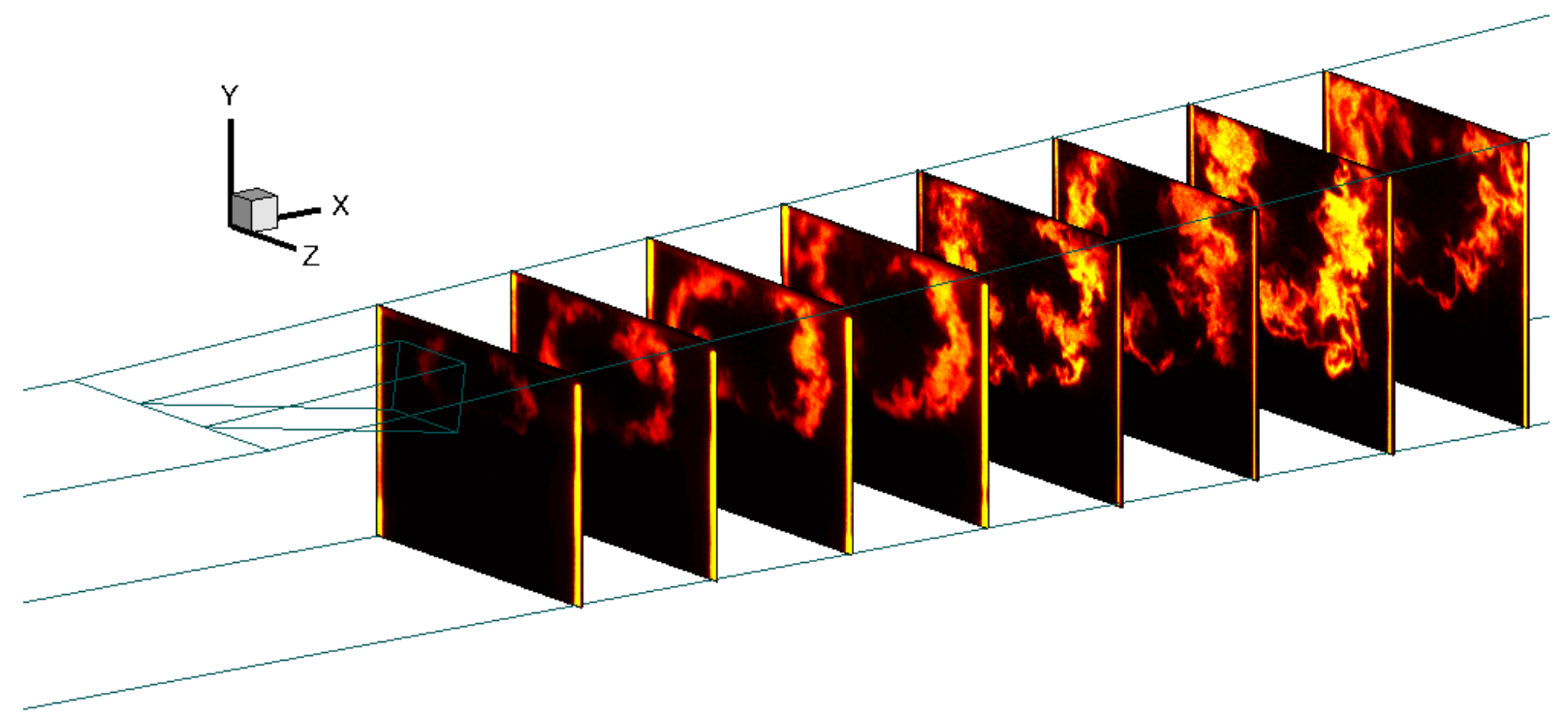

Figure 15: Streamwise distribution of PLIF overlaid onto wireframe drawing of combustor for $\phi=0.34$, scan from $x / H=22$ to 1.

A further effect of the equivalence ratio on the distribution of $\mathrm{OH}$ is shown in Fig. 16. Two values of $\phi$ were tested: 0.17 (right) and 0.34 (left). For a given mass flow rate of air, changes in the equivalence ratio are roughly proportional to changes in the concentration of $\mathrm{OH}$. As expected, with a higher fuel flow rate case (left), higher concentrations of $\mathrm{OH}$ result in a brighter PLIF signal intensity. At this streamwise position $(x / H=6)$, asymmetry is clearly evident for $\phi=0.17$, although some asymmetry is visible in the $\phi=0.34$ case as well. At further downstream positions, both equivalence ratio tests show symmetric distributions of $\mathrm{OH}$.

There are several mechanisms that can explain the asymmetry. It is possible that the flow diverges near the fuel injector creating a component of velocity in the direction of the laser sheet. If the laser was not spectrally centered on the $\mathrm{Q}_{1}(8)$ transition, then a Doppler shift would cause an asymmetric PLIF distribution. However, based on the cross-stream velocity components predicted by Fulton et al., this effect is expected to be negligible. ${ }^{15}$ Another 
possible mechanism to explain the flow asymmetry is laser absorption. As the laser sheet transfers energy through the excitation of the hydroxyl radicals, the laser energy diminishes across the test section, resulting in a decrease of fluorescence across the image. Laser absorption effects can be corrected assuming Beer's law of absorption, if the $\mathrm{OH}$ concentration distribution across the sheet is known. The direction of the laser sheet, however, is right-to-left in the image. The gradient in signal intensity is opposite to the laser direction, indicating that absorption is not a significant factor. Furthermore, with a higher equivalence ratio (Fig. 16, left image) it is expected that higher concentration levels of $\mathrm{OH}$ would amplify the laser absorption effect, which is clearly not observed from the results.

Flow asymmetry in the facility is the most likely explanation for the PLIF asymmetry. In a previous study by Cutler et al., CARS measurements of the temperature distribution for the given configuration showed significant temperature non-uniformities attributed to the heater, with higher temperatures recorded on the right side $(+z$ direction) of the facility upstream of the injector. ${ }^{14}$ It is possible that the asymmetric temperature distribution is one of the causes of the asymmetric PLIF signal distribution observed here. Radiative trapping, where fluorescence is reabsorbed by the highly populated vibrational ground state of $\mathrm{OH}$ at the excitation wavelength, is not likely a candidate to explain the observed asymmetry because radiative trapping would worsen with higher concentrations of $\mathrm{OH}$, which is opposite to the trend present in these results. Further experiments are required to determine the leading cause of this phenomenon.

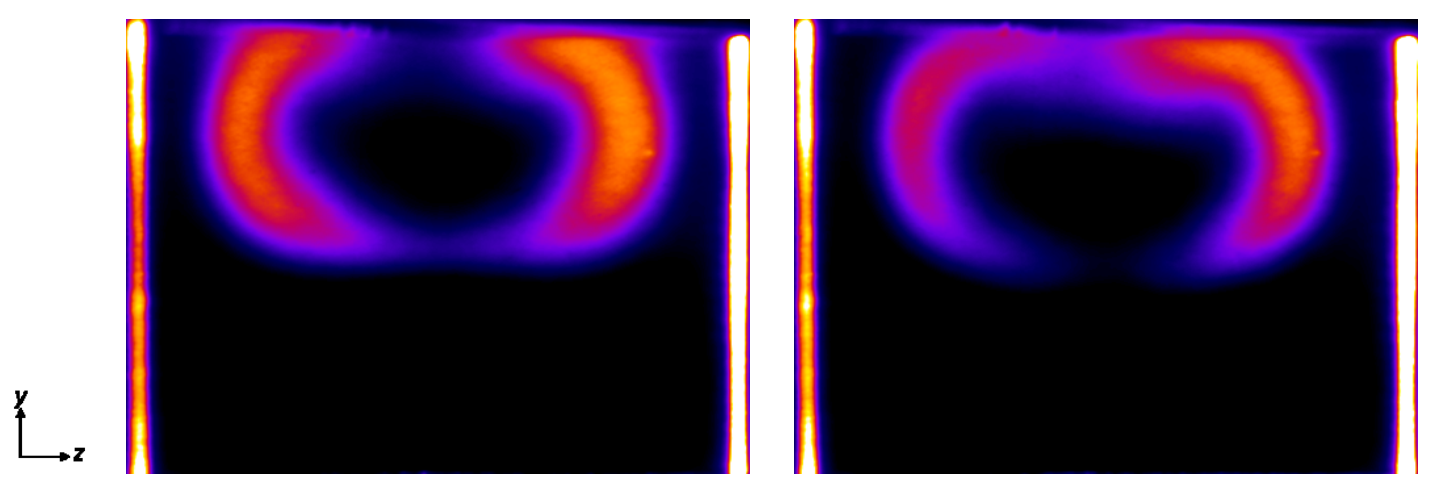

Figure 16: Effect of equivalence ratio; $\phi=0.34$ (left) and $\phi=0.17$ (right) at $x / H=6$; camera view 2 .

\section{Conclusions}

OH PLIF measurements in the combustor section of University of Virginia's dual-mode scramjet experiment have been performed. A detailed description of the post-processing, including the correction procedure for optical distortions, has been discussed. Visualization of the turbulent reaction zones corresponding to two equivalence ratio tests was achieved. Large scale turbulence structures are visible along the entire flame surface. Standard deviation plots reveal two bands indicating large fluctuation levels near the fuel-rich jet interior and near the fuel-lean jet exterior. Asymmetry near the fuel injector was observed for the lower equivalence ratio tests. Flow asymmetry due to non-uniform temperature distribution in the facility is likely the cause of this phenomenon. The results reported here serve as a dataset for numerical model assessment.

\section{Acknowledgements}

Dr. Johansen was supported by the Natural Sciences and Engineering Research Council of Canada (NSERC). This work was supported by the AFOSR/NASA National Center for Hypersonic Combined Cycle Propulsion grant FA 9550-09-1-0611. The technical monitors are Chiping Li from the AFOSR and Rick Gaffney from NASA.

\section{References}

${ }^{1}$ Fry, R.S., “A Century of Ramjet Propulsion Technology," Journal of Propulsion and Power, Vol. 20, No. 1, pp. 27-58, 2004.

${ }_{2}$ Tetlow, M.R., and Doolan, C.J., "Comparison of Hydrogen and Hydrocarbon-Fueled Scramjet Engines for Orbital Insertion,” Journal of Spacecraft and Rockets, Vol. 44, No. 2, pp. 365-373, 2007. 
${ }^{3}$ Smart, M.K., Hass, N.E., and Paull, A., "Flight data analysis of the HyShot 2 scramjet flight experiment" AIAA Journal, Vol. 44, No. 10, pp. 2366-2375, 2006.

4 Smart, M., "HiFiRE 7 - Development of a 3-D Scramjet for Flight Testing", AIAA-2009-7259, 16th AIAA/DLR/DGLR International Space Planes and Hypersonic Systems and Technologies Conference, Bremen, Germany, Oct. 19-22, 2009.

${ }^{5}$ Voland, R.T., Huebner, L.D., McClinton, C.R., "X-43A Hypersonic Vehicle Technology Development," Acta Astronautica, Vol. 59, pp. 181-191, 2006.

${ }^{6}$ Hank, J., Murphy, J., and Mutzman, R., "The X-51A Scramjet Engine Flight Demonstration Program", AIAA2008-2540, 15th AIAA International Space Planes and Hypersonic Systems and Technologies Conference, Dayton, Ohio, Apr. 28-1, 2008.

${ }^{7}$ Heiser, W.H., and Pratt, D.T., Hypersonic Airbreathing Propulsion. American Institute of Aeronautics and Astronautics, Inc., Washington, DC, 1994.

${ }^{8}$ Curran, E.T., "Scramjet Engines: The First Forty Years," Journal of Propulsion and Power, Vol. 17, No. 6, pp. 1138-1148, 2001.

${ }^{9}$ Forescue, P., and Stark, J., Spacecraft System Engineering, Wiley, New York, 1995.

${ }^{10}$ Fry, R.S., “A Century of Ramjet Propulsion Technology Evolution,” Journal of Propulsion and Power, Vol. 20, No. 1, pp. 27-58, 2004.

11 Rockwell, R.D., Goyne, C.P., Rice, B.E., Tatman, B.J., Smith, C., Kouchi, T., McDaniel, J.C., Fulton, J.A., and Edwards, J.R., "Close-Collaborative Experimental and Computational Study of a Dual-Mode Scramjet Combustor," AIAA-2012-113, 50th AIAA Aerospace Sciences Meeting and Exhibit, Nashville, TN, 2012.

${ }^{12}$ Goldstein, C.S., Shultz, I.A., Jeffries, J.B., and Hanson, R.K., "Tunable Diode Laser Absorption Sensor for Measurements of Temperature and Water Concentration in Supersonic Flows," AIAA-2011-1094, 49 ${ }^{\text {th }}$ AIA Aerospace Sciences Meeting and Exhibit, Orlando, FL, 2011.

${ }^{13}$ Busa, K.M., Bryner, E., McDaniel, J.C., Goyne, C.P., Smith, C.T., and Diskin, G.S., "Demonstration of Capability of Water Flux Measrment in a Scramjet Combustor using Tunable Diode Laser Absorption Tomography and Stereoscopic PIV," AIAA-2012-1294, 49 ${ }^{\text {th }}$ Aerospace Sciences Meeting and Exhibit, Orlando, FL, 2011.

${ }^{14}$ Cutler, A.D, Magnotti, G., Cantu, L., Gallo, E., Danehy, P.M., Rockwell, R., Goyne, C., McDaniel, J., "DualPump CARS Measurements in the University of Virginia's Dual-Mode Scramjet: Configuration A," AIAA-20120114, 50th AIAA Aerospace Sciences Meeting and Exhibit, Nashville, TN, 2012.

${ }^{15}$ Fulton, J.A., Edwards, J.R., Hassan, H.A., Rockwell, R., Goyne, C., McDaniel, J., Smith, C., Cutler, A., Johansen, C., Danehy, P.M., and Kouchi, T., "Large-Eddy / Reynolds-Averaged Navier-Stokes Simulations of a Dual-Mode Scramjet Combustor," AIAA-2012-0115, 50th AIAA Aerospace Sciences Meeting and Exhibit, Nashville, TN, 2012.

16 Goyne, C.P., Rodriguez, C.G., Krauss, R.H., McDaniel, J.C., and McClinton, C.R., "Experimental and Numerical Study of a Dual-Mode Scramjet Combustor," Journal of Propulsion and Power, Vol. 22, No. 3, pp. 481489, 2006

${ }^{17}$ O’Byrne, S., Stotz, I., Neely, A.J., Boyce, R.R., Mudford, N.R., and Houwing, A.F.P., “OH PLIF Imaging of Supersonic Combustion using Cavity Injection," AIAA-2005-3357, $13^{\text {th }}$ International Space Planes and Hypersonics Systems and Technologies Meeting, Capula, Italy, 2005.

18 Danehy, P.M., Alderfer, D.W., Inman, J.A., Berger, K.T., Buck, G.M., and Schwartz, R.J., "Fluorescence Imaging and Streamline Visualization of Hypersonic Flow over Rapid Prototype Wind-Tunnel Models," Proc. IMechE, Part G: J. Aerospace Engineering, 222(G5), 637-651 (2008)

19 Rasband, W.S., ImageJ, U.S. National Institutes of Health, Bethesda, MD, USA, http://rsb.info.nih.gov/ij, 1997-2009.

${ }^{20}$ Sanchez Sorzano, C.O., Thevenaz, P., and Unser, M., "Elastic Registration of Biological Images Using Vector-Spline Regularization," IEEE Transactions on Biomedical Engineering, Vol. 52, No. 4, 2005 , pp. $652-663$. 